\title{
On Duality, Symmetry and Symmetry lost in Solid Mechanics
}

\author{
Bui Huy Duong 1,2,3 \\ ${ }^{1}$ Laboratory of Solid Mechanics, UMR X-CNRS 7649, Ecole Polytechnique ParisTech, Palaiseau, \\ ${ }^{2}$ LaMSID/R\&D/UMR EDF-CNRS 2832, Clamart, France, \\ ${ }^{3}$ UME/ENSTA ParisTech, Palaiseau, France. \\ e-mail: hdb@1ms.polytechnique.fr
}

\begin{abstract}
The paper recalls the concept of duality in Mathematics and extends it to Solid Mechanics. One important application of duality is to restore some symmetry between current fields and their adjoint ones. This leads to many alternative schemes for numerical analyses, different from the classical one as used in classical variational formulation of boundary value problems (Finite Element Method).

Usually, Conservation laws in Fracture Mechanics make use of the current fields, displacement and stress. Many conservation laws of this type are not free of the source term. Consequently, one cannot derive path-independent integrals for use in Fracture Mechanics.

The introduction of variables and dual or adjoint variables leads to true path-independent integrals. Duality also introduces some anti-symmetry in current fields and adjoint ones for some boundary value problems. The symmetry is lost between fields and adjoint fields. The last notion enables us to derive new variational formulation on dual subspaces and to exactly solve inverse problems for detecting cracks and volume defects.
\end{abstract}

Key words: Duality, Symmetry lost, Inverse Problems

\section{INTRODUCTION}

This paper makes a review of some recent works of the author and of his research groups on these topics. The central theme Duality is not a new concept since it was known in Analytical Mechanics, since Lagrange two and half centuries ago. Today in Engineering Mechanics, Duality is widely used in the formulation of Boundary Values Problems (BVP) in Elasticity, Plasticity etc using test functions. If we consider dual variables, for example stress $\sigma$ and strain $\varepsilon$ or strain rate $\varepsilon^{*}$, their duality can be defined by the energy $W=\frac{1}{2} \sigma: \varepsilon$ or the virtual power $\sigma: \varepsilon^{*}$ respectively. The concept of virtual power, introduced by P. Germain [1] for the Mechanics of continuous media, is crucial in modern Computational Mechanics by considering the variational formulation of BVP, instead of the field equations and boundary conditions themselves. It originates from the mathematical concept of spaces and dual spaces culminating in the theory of Distribution of L. Schwartz [2] (for which he was awarded the Fields medal, 1951). Using these concepts, P. Germain viewed the stress $\sigma$ as an element of the space D' which is dual to the strain rate space $\mathrm{D}$ of elements $\varepsilon^{*}$. As illustrated below, duality reveals some symmetry between these spaces, independently of another physical symmetry existing in materials (for example symmetrical moduli tensor in elasticity).

When the constitutive law has a material symmetry, which is the case of classical Elasticity, the overall response of a solid exhibits a symmetry, as observed in the Betti-Somigliana identity. 
What happens when we consider two elastic solids having the same external geometry for which the overall responses do not have symmetry property? We can say that there is a Symmetry lost between two states, one for the sound body and another one for a body with internal defects. It is simply due to the fact Betti-Somigliana's identity fails in this case. The aim of this paper is to show that duality and symmetry lost are the right tools to solve exactly some non-linear inverse problems, while conventional methods of inverse problems, based of parameter optimization, can only provide approximate solutions.

\section{DUALITY AND SYMMETRY}

We quote P. Germain "Force is the dual of mobility (velocity)". It belongs to the dual space V' of the space $\mathrm{V}$ of velocity fields. There are other dual variables or fields as shown in Table I where operators and adjoint operators acting on dual elements are also listed.

Table I: Examples of duality

\begin{tabular}{|c|c|c|c|}
\hline Variables or functions & Action or results & $\begin{array}{l}\text { Dual variables and adjoint } \\
\text { functions }\end{array}$ & Remarks \\
\hline Displacement $\mathbf{u}$ & Work & Force $\mathbf{f}$, Traction $\mathbf{T}$ & \\
\hline Velocity $\mathbf{v}^{*}$ & Virtual power & Force $\mathbf{f}$, Traction $\mathbf{T}$ & \\
\hline Strain rate $\boldsymbol{\varepsilon}^{\prime}$ & Power & Stress $\sigma$ & \\
\hline Potencial energy $\mathrm{P}(\boldsymbol{\varepsilon}(\mathbf{u}))$ & Elasticity & Complementary potencial $\mathrm{Q}(\boldsymbol{\sigma})$ & \\
\hline J-integral (Rice) & Fracture mechanics & Dual I-integral & \\
\hline Projection $\mathrm{P}$ & Tomography & Back projection $\mathrm{P}^{*}$ & $\begin{array}{l}\mathrm{P}, \mathrm{P}^{*} \text { used in } \\
\text { Radon's transform }\end{array}$ \\
\hline Propagation of waves & Elastic scattering & Back propagation of waves & \\
\hline $\begin{array}{l}\text { Forward equations } \\
\text { Forward problems }\end{array}$ & $\begin{array}{l}\text { Reciprocity gap } \\
\text { functional }\end{array}$ & $\begin{array}{l}\text { Adjoint equations } \\
\text { Inverse problems }\end{array}$ & \\
\hline Primal problems & Convex analysis & Dual problems & \\
\hline Schwartz's spaces D, S & Distribution theory & Dual spaces D', S' & \\
\hline Sobolev's space $\mathrm{H}^{\mathbf{m}, \mathbf{p}}$ & Functional analysis & Dual space $\mathrm{H}^{-\mathbf{m}, \mathbf{p}}$ & $1 / \mathrm{p}+1 / \mathrm{p}^{\prime}=1$ \\
\hline
\end{tabular}

To better see the structure of elasticity, let us consider the Tonti's diagram in the quasi-static case [3]. The displacement method of solving the equilibrium equation in elasticity is given by the following maps:

$$
\mathbf{u} \rightarrow \varepsilon=\frac{1}{2}\left(\nabla+\nabla^{t}\right) \mathbf{u} \rightarrow \sigma=L \varepsilon \rightarrow-\operatorname{div} \sigma=0 \text { (equilib .) }
$$

Operator $D=-d i v$ and $D^{*}=\frac{1}{2}\left(\nabla+\nabla^{t}\right)$ are adjoint to each other, $L$ is the elastic moduli tensor. The stress method is described by the following maps:

$$
B \rightarrow \sigma=R R^{*} B \rightarrow \varepsilon=L^{-1} \sigma \rightarrow R R^{*} \varepsilon=0 \quad \text { (compatib.) }
$$

where $B$ is the Beltrami symmetric second order tensor, $R$ is the right curl operator defined by $R B:=-\left(\partial_{k} B_{i j}\right) \mathbf{e}^{i} \otimes\left(\mathbf{e}^{j} \wedge \mathbf{e}^{k}\right)$ and $R^{*}$ is the adjoint left curl operator defined by $R^{*} B:=$ $\left(\partial_{k} B_{i j}\right)\left(\mathbf{e}^{k} \wedge \mathbf{e}^{i}\right) \otimes \mathbf{e}^{j}$. Operator RR* is self-adjoint. In two-dimensions, $B=\psi\left(x_{1}, x_{2}\right) \mathbf{e}^{3} \otimes \mathbf{e}^{3}$, where $\psi\left(x_{1}, x_{2}\right)$ is the Airy's function. The Tonti diagram has been generalized to elastodynamics [9], as shown in Figure 1. We remark that operators $\mathrm{C}$ and $\mathrm{C}^{*}$ are adjoint to each 
other, as well as operators $\mathrm{S}$ and $\mathrm{S}^{*}$. The elastodynamic diagram reveals the profound symmetry of equations independently of the physics of the body.

The displacement method of linear elasticity is described by Figure 2 .

The stress method of linear elasticity is described by Figure 3.

The hybrid method of Ladeveze [4] also called the error in constitutive law method is described by Figure 4.

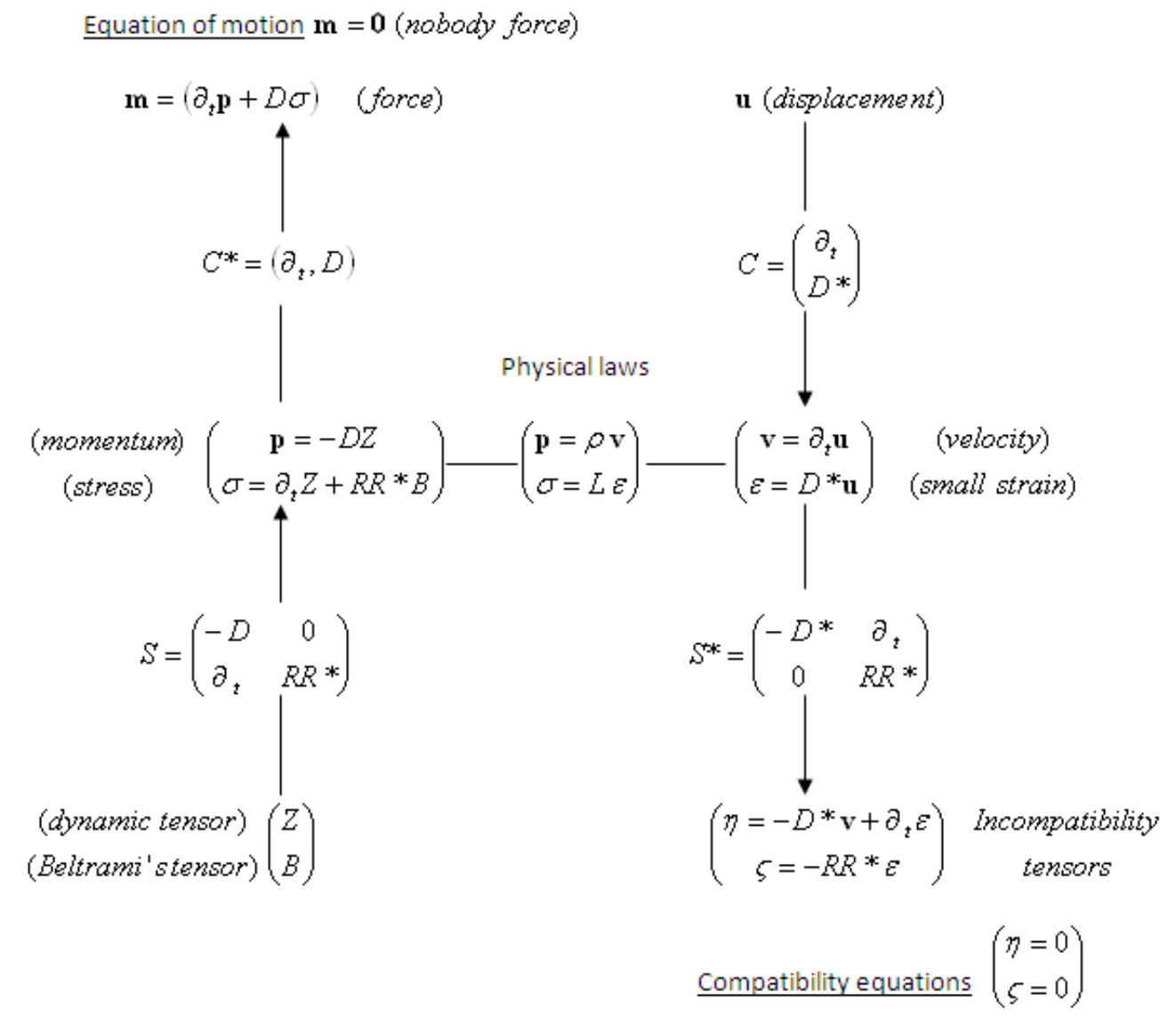

Figure 1: Generalized diagram of Elastodynamics which shows the symmetry between dynamics and kinematics chains, described in opposite directions [9]. The physical law is not necessarily linear in this diagram. 
Equation of motion $\mathbf{m}=\mathbf{0}$ (nobody force)

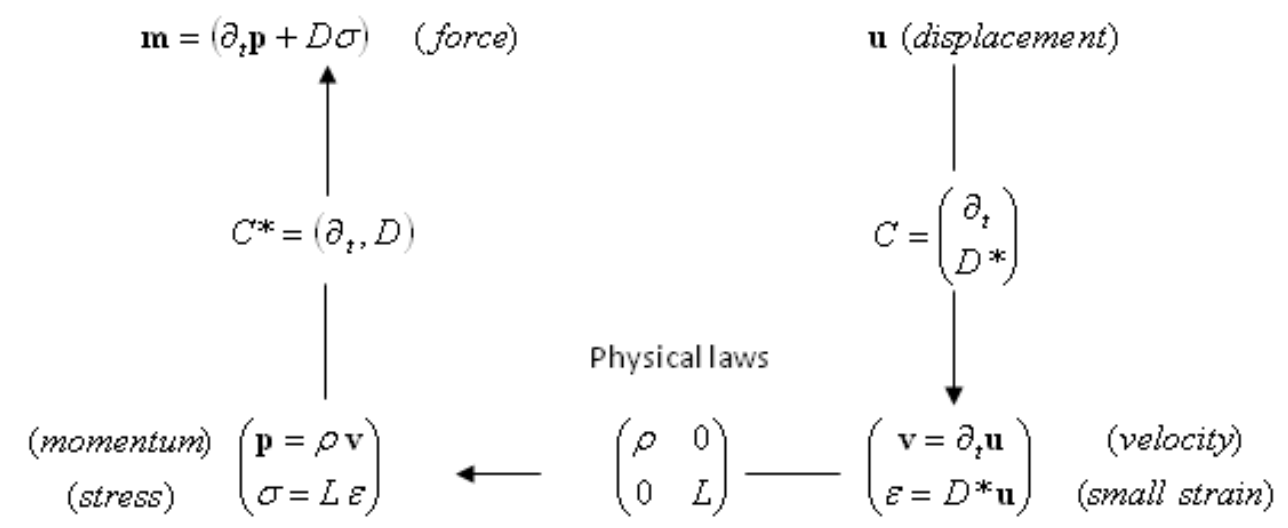

Figure 2: The displacement finite elements method based on the equation of motion..

Physical laws

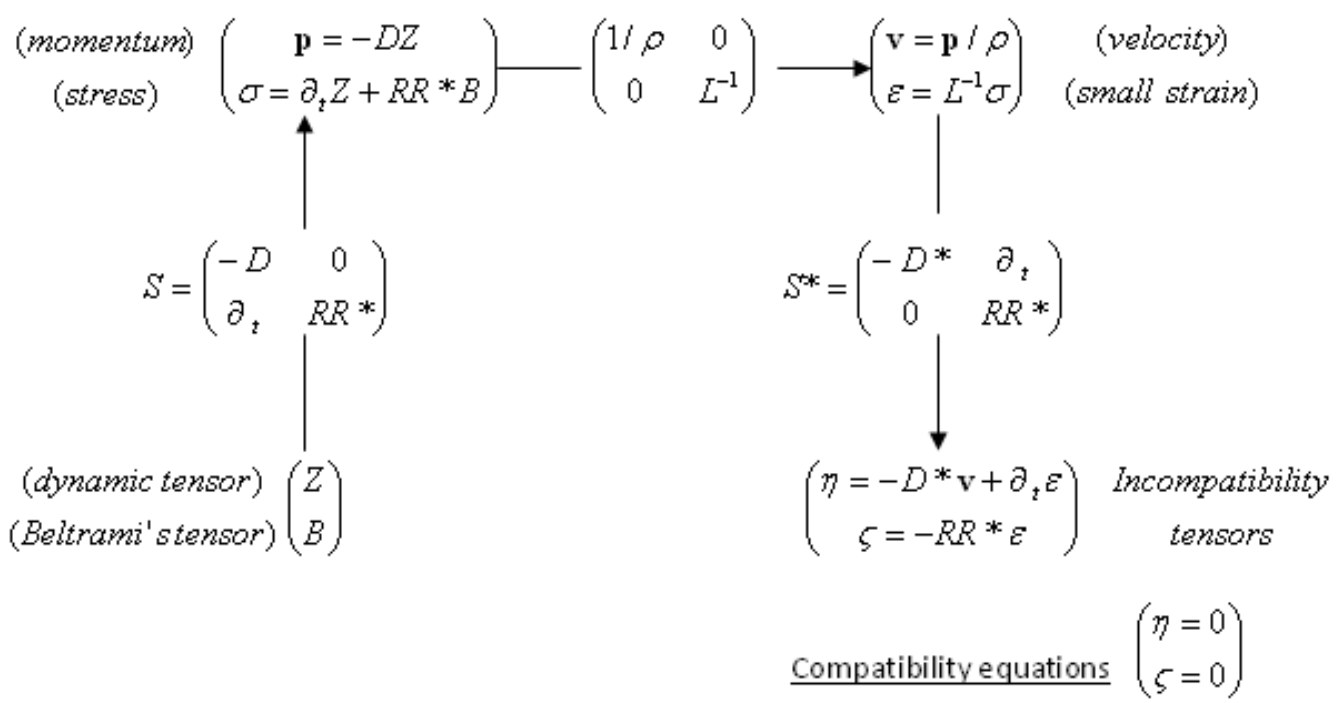

Figure 3: The stress finite elements method starts from tensors (Z, B) considered as unknowns for which the equation of motion is satisfied. One has only to express the compatibility equations. 


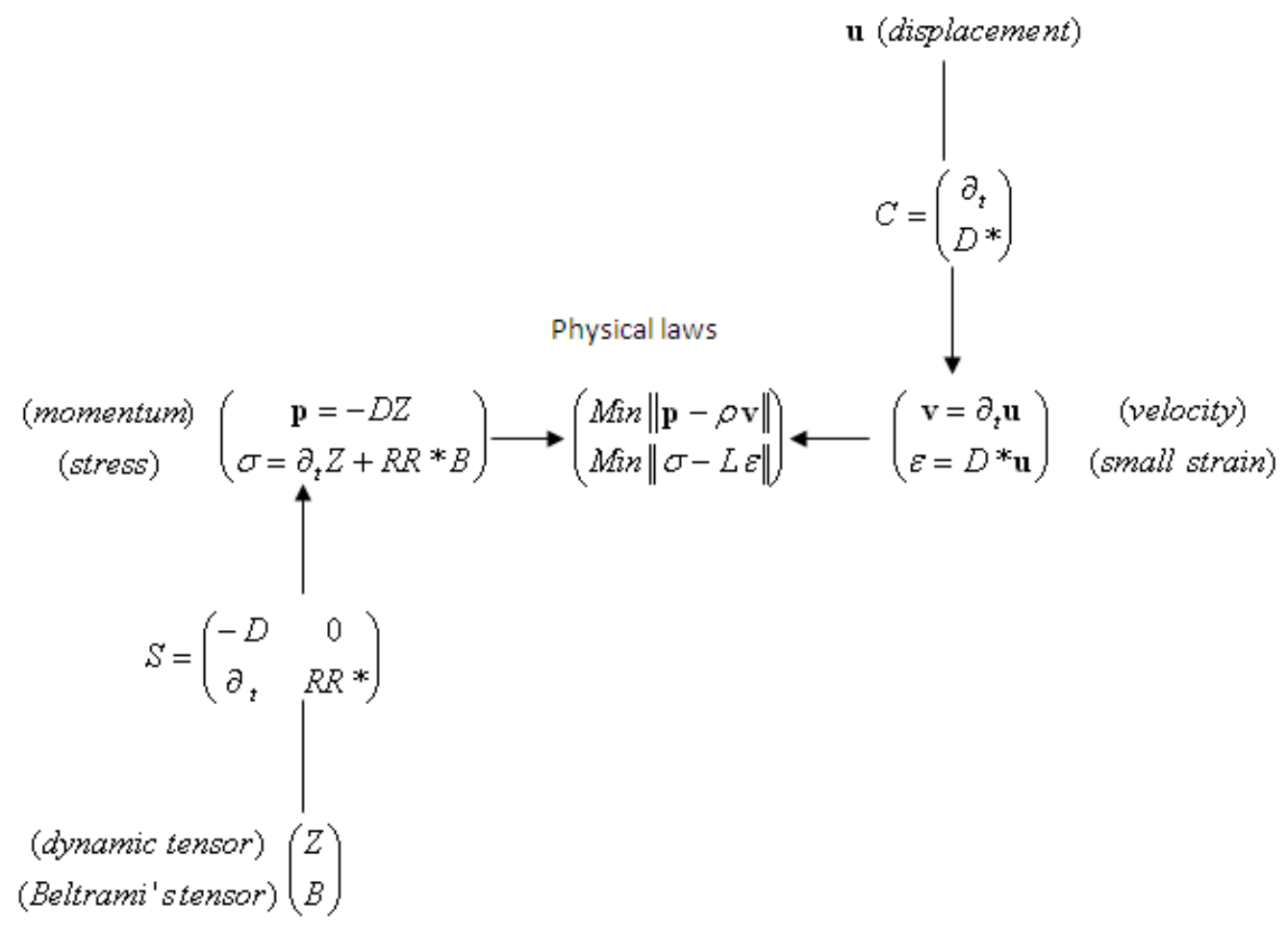

Figure 4: The hybrid finite elements method, also called the "error in constitutive laws" method, consists in the matching of the kinematical scheme with the dynamical one, in such a way that the norm of the error in constitutive laws is minimal [4].

Remarks: In statics, there are many variational formulations of boundary value problems of elassticity. The displacement method consists of considering the kinematic chart: $\mathbf{u} \rightarrow$ $\varepsilon(\mathbf{u})=\mathrm{D}^{*} \mathbf{u} \rightarrow \sigma=\mathrm{L}(\varepsilon)$ of Figure 2 and the minimization of the potential energy $\mathrm{P}(\mathbf{u})$, with $\mathbf{u} \in \mathbf{K}$ in the kinematically admissible ensemble $K$ such that $\mathbf{u}=\mathbf{u}^{\mathrm{d}}$. The solution of the minimization problem satisfies the equelibrium equation $\mathrm{D} \sigma=0$ (no body force). The stress method consists of considering, Figure 3, the chart: $\sigma$ (subject to $D \sigma=0$ in $\Omega$ and $\sigma \cdot \mathbf{n}=\mathbf{T}^{\mathrm{d}}$ on $\left.\mathrm{S}^{\mathrm{T}}\right) \rightarrow \varepsilon=\mathrm{L}^{-1}(\sigma)$ and the minimization of the complementary potential $\mathrm{Q}(\sigma)$. The solution $\varepsilon(\sigma)$ is compatible.

Let us mention the mixed method, consisting in the minimization of the complementary potential $\mathrm{Q}\left(\mathrm{LD}^{*} \mathbf{u}\right)$, expressed with displacement variable $\mathbf{u}$, satisfying the equilibrium equation DLD* $\mathbf{u}=0$, in $\Omega$ (no body force) and the stress boundary condition $L D^{*} \mathbf{u} \cdot \mathbf{n}=\mathbf{T}^{\mathrm{d}}$ on $\mathrm{S}^{\mathrm{T}}$. The solution $\mathbf{u}$ is found to satisfy the displacement boundary condition on $S^{\mathbf{u}}$, i.e $\mathbf{u} \in \mathbf{K}$.

\section{CONSERVATION LAWS}

Conservation laws of the type $-\operatorname{div} a+b=0$ are found in Solid Mechanics. For example, the equation of motion is the conservation law of the momentum $-\operatorname{div} \sigma+\rho \partial_{t} \partial_{t} \mathbf{u}=0$. In Fracture Mechanics, conservation laws of the pure divergence form - diva $=0$, without source terme $b$, are at the origin of path-independent integrals which are very useful for obtaining crack tip parameters. Most conservation laws of this type have an energetic interpretation. They are 
derived from the Noether's theorem of invariance of the Lagrangian for the symmetry group of translation, in homogeneous body. The conservation laws of the energy-momentum given by Eshelby (1951) in elastostatics, or Fletcher (1976) in elasto-dynamics are indicated respectively by the following equations, where $W(\varepsilon)$ is the strain energy density

$$
\begin{gathered}
a=W(\varepsilon) I-\left(\operatorname{grad}^{t} \mathbf{u}\right) \cdot \sigma, \quad b=0 \quad(\text { Eshelby }) \\
\left.a=W(\varepsilon) I-\frac{1}{2} \rho \dot{\mathbf{u}}^{2} I-\left(\operatorname{grad}^{t} \mathbf{u}\right) \cdot \sigma, \quad b=\frac{\partial}{\partial t}(\rho \dot{\mathbf{u}} . \operatorname{grad} \mathbf{u}) \quad \text { (Fletcher }\right)
\end{gathered}
$$

Eshelby's law without source term leads to Rice's path-independent J-integral in elasto-statics along a contour $\Gamma$ around the crack tip

$$
J=\int_{\Gamma}\left[W(\varepsilon) n_{1}-(\sigma . \mathbf{n}) \cdot \mathbf{u}{ }_{, 1}\right] d s
$$

while with Fletcher's conservation law ones has an additional area integral over the crack tip region

$$
J^{d y n}=\int_{\Gamma}\left[W(\varepsilon) n_{1}-\frac{1}{2} \rho \dot{\mathbf{u}}^{2} n_{1}-(\sigma \cdot \mathbf{n}) \cdot \mathbf{u}{ }_{, 1}-\rho \dot{\mathbf{u}} \cdot \mathbf{u}_{, 1}\right] d s+\frac{d}{d t} \int_{A(\Gamma)} \rho \dot{\mathbf{u}} \cdot \mathbf{u}, 1, d A
$$

$J^{d y n}$ has the meaning of the derivative of the energy with respect to the crack propagation rate. Its value in mode $I$ is $J^{d y n}=\left(1-v^{2}\right) K_{I}^{2} f_{I}(V) / E$ where $f_{I}(V)$ is an universal function of the velocity $\mathrm{V}, v$ is the Poisson ratio, $\mathrm{E}$ is the Young modulus and $K_{I}$ is the stress intensity factor, Achenbach [14].

Does a true path-independent integral exist in elastodynamics?

This question was solved by considering duality and adjoint variables. Together with the current elasto-dynamical field $\mathbf{u}(\mathbf{x}, \mathrm{t})$, from which stress and strain are derived, an adjoint field $\mathbf{v}(\mathbf{x}, \mathrm{t} ; \tau)$ satisfying the same elasto-dynamical equation $-\operatorname{div} \sigma[\mathbf{v}]+\rho \partial_{t} \partial_{t} \mathbf{v}=0$, with final conditions, where $\tau$ is a parameter

$$
\mathbf{v}(\mathbf{x}, t ; \tau)=0, \quad \text { for } t>\tau
$$

The true path independent integral is

$$
H=\frac{1}{2} \int_{\Gamma} \int_{0}^{\tau}\{\mathbf{n} \cdot \sigma[\mathbf{u}] \mathbf{v}-\mathbf{n} \cdot \sigma[\mathbf{v}] . \mathbf{u}\} d s d t
$$

has the following property: $H(\mathbf{u}, \mathbf{v})=\left(1-v^{2}\right) K_{I}(\mathbf{u}) * K_{I}(\mathbf{v}) / E$ where the symbol (*) is the time convolution. Using (8) one sees that the stress-intensity factor $K_{I}(\mathbf{u})$ can be determined from boundary data, and known stress-intensity factor of the adjoint field $K_{I}(\mathbf{v})$, by solving a time deconvolution linear problem [8]. Path independent integral H, Eq. (8), reveals some symmetry (or rather an anti-symmetry) between current field $\mathbf{u}$ and its adjoint $\mathbf{v}$. 


\section{SYMMETRY LOST AND INVERSE PROBLEMS FOR CRACK DETECTION}

In the previous section, when dealing with propagating waves $\mathbf{u}$ and back propagating waves $\mathbf{v}$, we found an anti-symmetry between current $\mathbf{u}$ and adjoint fields $\mathbf{v}$, in the derivation of pathindependent integral of the form, with an arbitrary path $\Gamma$ around the crack tip

$$
H=\frac{1}{2} \int_{\Gamma} \int_{0}^{\tau}\{\mathbf{n} \cdot \sigma[\mathbf{u}] . \mathbf{v}-\mathbf{n} \cdot \sigma[\mathbf{v}] . \mathbf{u}\} d s d t>0
$$

In quasi-static elasticity, one has the energy release rate $G>0$, denoted here by $R$

$$
R=\frac{1}{2} \int_{\Gamma}\{\mathbf{n} \cdot \sigma[\mathbf{u}] \mathbf{v}-\mathbf{n} \cdot \sigma[\mathbf{v}] . \mathbf{u}\} d s
$$

We say that there is a symmetry lost between current field $\mathbf{u}$ and adjoint field $\mathbf{v}$. Symmetry lost occurs when current field corresponds to a solid with defects (cracks, inclusion etc) while adjoint field corresponds to arbitrary fields in a sound solid. We have $R=0$ when the current field correspond to the sound solid too, and $R \neq 0$ when there is a defect in the solid. Therefore, $R$ has not the thermodynamic interpretation of the energy release rate $\mathrm{G}$ and becomes a defect indicator

$$
\left\{\begin{array}{llr}
R=0 & \Leftrightarrow & \text { No defects inside the solid } \\
R \neq 0 & \Leftrightarrow & \text { Existence of } a \text { defect }
\end{array}\right.
$$

The method of solution reduces to the search of the zeros of a functional, or simply the zeros of a function of defect parameters. The arbitrariness of the choice of adjoint fields is crucial for the derivation of the solutions to some crack inverse problems, using boundary data. A series of recent papers showed that the reciprocity gap functional $R$ provided closed form solutions to many inverse crack problems, for electrostatics [13], static elasticity [11], diffusion equation [12], transient acoustics [15] and elasto-dynamics, with the exact solution to an earthquake inverse problem [16], [17]. These solutions can be found in [10], [19]

In what follows, we consider an inverse crack problem in elasto-dynamics, Fig. 1, which modelizes an earthquake. Earthquake results from the sudden release of stresses in a planar fault $\Sigma(\mathrm{t})$, which is generally a time dependent surface, originating at points of highest shear stress. Both the fault surface $\Sigma(\mathrm{t})$ and the stress release on its plane are unknown. The data for the inversion consist of the measurement of the acceleration, hence by a twice time integration, the displacement field $\mathbf{u}(\mathbf{x}, \mathrm{t})$ of points on the external boundary $\mathrm{S}_{\mathrm{ext}}$,

This elasto-dynamic inverse problem is generally solved by methods of minimization of the residual, which is the norm in space-time of the difference between a theoretical prediction of the surface displacement field and the measured one on the boundary. Such methods based on the best fitting of data are essentially numerical and, more importantly, cannot provide exact solutions particularly in space-time domain. A statement on the impossibility of an exact 
solution, in elasto-dynamics, was given by Das and Suhadolc [18] "..even if the fitting of data seems to be quite good, the faulting process is poorly reproduced, so that in the real case, it would be difficult to know when one has obtained the correct solution".

Now, we show that the reciprocity gap functional method, which can be considered as a special form of the variational method, using sub-spaces of adjoint functions, provides us the exact solution to the earthquake inverse problem. We illustrate the method by an example of crack inverse problem for the heat equation given in [12], for which $\mathrm{u}$ and $\mathrm{v}$ are scalar fields. We assume that there is a stationary planar crack.

The key method of solving inverse problems relies on the appropriate choices of sub-spaces of adjoint functions $\mathrm{v}$ which are related to cracks parameters. Consider the heat diffusion equation (forward problem):

$$
\begin{gathered}
\partial_{t} u-\Delta u=0, \quad \mathbf{x} \in(\Omega-\Sigma) \times[0, T] \\
u(\mathbf{x}, 0)=0, \quad \text { in }(\Omega-\Sigma) \\
u(\mathbf{x}, t)=u^{d}, \quad \mathbf{x} \in S_{e x t} \quad \text { (measured data) } \\
\partial_{n} u(\mathbf{x}, t)=\Phi \quad \quad \mathbf{x} \in S_{\text {ext }} \text { (measured data) } \\
\partial_{n} u(\mathbf{x}, t)=0, \quad \mathbf{x} \in \Sigma \quad \text { (a priori knowledge) }
\end{gathered}
$$

The adjoint equation (backward diffusion problem) for an uncracked body :is

$$
\begin{gathered}
\partial_{t} v+\Delta v=0, \quad \mathbf{x} \in \Omega \times[0, T] \\
v(x, T)=0, \quad \mathbf{x} \in \Omega
\end{gathered}
$$

Combining these equations, we obtain a non-linear variational equation

$$
\int_{0}^{T} \int_{\Sigma(u)}[[u]] \partial_{n} v d S d t=\int_{0}^{T} \int_{S e x t}\left(\Phi v-u^{d} \partial_{n} v\right) d S d t:=R(v), \quad \forall v
$$

The non-linearity comes from the unknown integration domain $\Sigma(\mathrm{u})$, in the left hand side of the equation. The reciprocity gap $\mathrm{R}$ depends only on the surface data and the adjoint function.

There are three unknowns: 1 . the normal to the crack plane; 2 . the position of the crack plane; 3 . the geometry of the crack.

\section{Determination of the normal}

The normal $\mathbf{n}$ to the crack plane can be firstly determined by using the subspace $\mathrm{V}_{1}$ of adjoint functions parameterized by vector $\mathbf{p}$, in the form ( $\mathrm{erf}$ : error function) 


$$
v(\mathbf{x})^{(p)}=\left\{\begin{array}{lc}
1-\operatorname{erf}\left(\frac{\mathbf{x} \cdot \mathbf{p}}{2 \sqrt{T-t}}\right) & (t \leq T) \\
0 & (t>T)
\end{array}\right.
$$

By taking $\mathbf{p}$ in the form $\mathbf{p}=\mathbf{n} \times \mathbf{m}$, we verify that the normal $\mathbf{N}$ is the solution of a MinMax problem

$$
\mathbf{N}=\arg \operatorname{Min}_{\|n\|=1}\left\{\operatorname{Max}_{\|\mathrm{m}\|=1, \mathbf{n} . \mathbf{m}=0} R(\mathbf{n} \times \mathbf{m})\right\}
$$

\section{Determination of the crack plane}

We consider the subspace $\mathrm{V}_{2}$ of adjoint functions parameterized by the scalar $\mathrm{c}$ in the form

$$
v(\mathbf{x})^{(c)}=\frac{1}{\sqrt{4 \pi(T-t)}} \exp \left\{\frac{-\left(x_{3}-c\right)^{2}}{4(T-t)}\right\}, \quad t<T
$$

$v(\mathbf{x})^{(c)}=0, t>T$, where $\mathrm{Ox}_{3}$ is directed along the normal $\mathbf{N}$. Let the position of the crack plane be defined by $\mathrm{x}_{3}-\mathrm{C}=0$. It can be checked that the function $\mathrm{c} \rightarrow \mathrm{R}\left(\mathrm{v}^{(\mathrm{c})}\right)$ has an unique zero at $\mathrm{c}=\mathrm{C}$. Therefore, the transition of $\mathrm{R}\left(\mathrm{v}^{(\mathrm{c})}\right)$ from its non zero value $\mathrm{R}\left(\mathrm{v}^{(\mathrm{c})}\right) \neq 0$ to $\mathrm{R}\left(\mathrm{v}^{(\mathrm{c})}\right)=0$ determines the position $\mathrm{C}$ of the crack plane. We have an example of the zero-crossing method to solve an inverse problem.

\section{Determination of the crack geometry}

Having determined the crack plane, taken as the plane $\mathrm{Ox}_{1} \mathrm{x}_{2}$, we determine the crack geometry as the support of the crack discontinuity $\Sigma=\operatorname{supp}\{[[\mathrm{u}]]\}$. We introduce the subspace $\mathrm{V}_{3}$ of adjoint function parameterized by $\left(\mathrm{s}_{1}, \mathrm{~s}_{2}, \mathrm{q}\right) \in \mathrm{R}^{3}, \mathrm{q}=\mathrm{q}_{\mathrm{R}}+\mathrm{iq}_{\mathrm{I}}, \mathrm{q}_{\mathrm{I}}>0$, defined by

$$
v(\mathbf{x})^{\left(s_{1}, s_{2}, q\right)}=\exp (i q t) \exp \left\{-i\left(s_{1} x_{1}+s_{2} x_{2}\right)\right\} \exp \left\{x_{3}\left(s_{1}^{2}+s_{2}^{2}-i q\right)^{1 / 2}\right\}, \quad t<T
$$

and $v(\mathbf{x})^{\left(s_{1}, s_{2}, q\right)}=0$, for $\mathrm{t}>\mathrm{T}$. Let $\mathrm{D}(\mathbf{x}, \mathrm{t})=[[\mathrm{u}]]$ in $\Sigma, \mathrm{D}(\mathbf{x}, \mathrm{t})=0$ for $\mathbf{x}$ in the crack plane, outside $\Sigma$. Eq. (18) becomes

$$
\int_{R^{2}} H(\mathbf{x}, q) \exp \{-i(\mathbf{s . x})\} d^{2} x=\frac{1}{\sqrt{s_{1}^{2}+s_{2}^{2}-i q}} \int_{[0, \infty]} d t \int_{S_{e x t}}\left(u^{d} \partial_{n} v^{(\mathbf{s}, q)}-v^{(\mathbf{s}, q)} \Phi\right) d S:=h(\mathbf{s}, q)
$$

where the right hand side $\mathrm{h}(\mathrm{s}, q)$ of Eq. (23) is a known from boundary data and is a smooth function of $\mathbf{s}, q$ and 


$$
H(\mathbf{x}, q)=\int_{0}^{\infty} D(\mathbf{x}, t) \exp (-i q t) d t
$$

It has been shown in [12] that the spatial supports of functions $\mathbf{x} \rightarrow \mathrm{D}(\mathbf{x}, \mathrm{t})$ and $\mathbf{x} \rightarrow \mathrm{H}(\mathbf{x}, q)$, with $\mathrm{H}(\mathbf{x}, q)$ defined as the time Fourier transform of $\mathrm{D}(\mathbf{x}, \mathrm{t})$, are identical. It is proved that condition $\mathrm{q}_{\mathrm{I}}>0$ ensures the compactness of the supports of functions. Therefore the crack geometry is explicitly determined by the geometrical support of $\mathrm{H}(\mathbf{x}, \mathrm{q})$ given by the inverse spatial Fourier transform of $\mathrm{h}(\mathbf{s}, q)$

$$
\Sigma=\operatorname{supp}\left[F_{(x)}^{-1} h(\mathbf{s}, q)\right]
$$

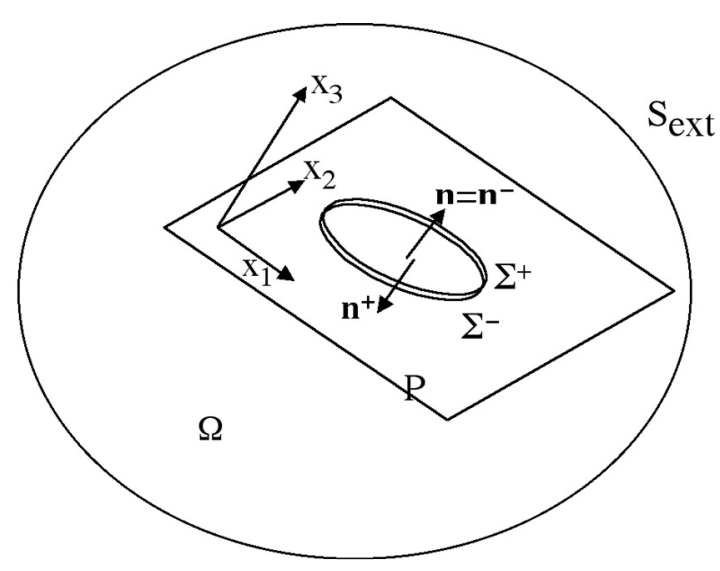

Figure 1: Geometry of the domain $\Omega$ and the unknown planar $\operatorname{crack} \Sigma$.

\section{REFERENCES}

[1] P. Germain. The method of virtual power in continuum mechanics. Part II, in Applications to Continuum Thermodynamics J.J.D Domingos et al (Eds); Wiley, NY, 1973; 317-333..

[2] L. Schwartz. Théorie des distributions. Hermann Paris, 1978.

[3] E. Tonti. On the formal structure of physical theories. Cooperative Library Instituto di Politecnico di Milan, 1975.

[4] P. Ladeveze. Comparison of models of continuum media. PhD Thesis Univ. Paris, 1975.

[5] H.D. Bui. A path independent integral for mixed mode of fracture in linear thermoelasticity. In IUTAM Symp. Fundamental of Deformation and Fracture, Sheffield April $1984 ; 597$.

[6] J.D. Eshelby. The force on an elastic singularity. Phil. Trans. Roy. Soc. London, 1951; A 244; 87-112.

[7] D. C. Fletcher. Conservation laws in linear elasto-dynamics. Arch. Rat. Mech. Anal., 1976; 60; p. 329.

[8] H.D. Bui and H. Maigre. Extraction of stress-intensity factors from global mechanical 
quantities. C.R. Acad. Sci. Paris, 1988; 306: p.1213.

[9] H.D. Bui. On the variational boundary integral equations in elasto-dynamics with the use of conjugate functions, J. Elasticity, 1992; 28; 247.

[10] H.D. Bui. Fracture Mechanics: Inverse Problems and Solutions. Springer, 2006. Vietnamese translation, NXBKH\&CN Hanoi 2008; Russian translation, Fyzmalit Moscow 2011.

[11] S. Andrieux, A. Ben Abda and H.D. Bui. Reciprocity principle and crack identification. Inverse Problems, 1999; 15; 59-65.

[12] A. Ben Abda and H.D. Bui. Reciprocity and crack identification in transient thermal problems. J. Inv. Ill-posed Problems, 2001; 9; 1-6.

[13] S. Andrieux and A. Ben Abda. Identification de fissures planes par une donnee de bord unique: un procede direct de localisation et d'identification. C.R. Acad. Sci. Paris, 1992, 1 (315); 1323-1328.

[14] J.D. Achenbach. Wave propagation in elastic solids. North Holland Amsterdam, 1980.

[15] H. D.Bui, A. Constantinescu and H. Maigre. Inverse scattering of a plananr crack in 3D acoustics: close form solution for a bounded solid. C.R. Acad. Sci. Paris, 1999; 327; 91976.

[16] H. D.Bui, A. Constantinescu and H. Maigre. An exact inversion formula for determining a planar fault from boundary measurements. J. Inv. Ill-posed Problems, 2005; 13(6); 553565.

[17] H. D.Bui, A. Constantinescu and H. Maigre. Numerical identification of linear cracks in 2D elastodynamics using the instantaneous reciprocity gap. Inverse Problems; 2004; 20; 993-1001.

[18] S. Das and P. Suhadolc. On the inverse problem for earthquake rupture. The Haskell-type source model. J. Geophys. Res., 1996; 101(B3); 5725-5738.

[19] A. Ehrlacher and X. Markenscoff. Duality, Symmetry and Symmetry lost in Solid Mechanics. Selected works of H.D. Bui; Presses des Ponts, Paris, 2011. 